\title{
Crabrolin, a natural antimicrobial peptide: structural properties.
}

Massimiliano Aschi ${ }^{\mathrm{a}^{*}}$, Argante Bozzi $^{\mathrm{b}}{ }^{\circledR}$, Carla Luzi $^{\mathrm{b}}$, Nadia Bouchemal $^{\mathrm{c}}$ and Marco Sette $^{\mathrm{d}}$

${ }^{a}$ Department of Physical and Chemical Sciences, University of L'Aquila. Via Vetoio 67100 L'Aquila - Italy

${ }^{\mathrm{b}}$ Department of Biotechnological and Clinical Sciences, University of L'Aquila Via Vetoio 67100 L'Aquila - Italy

${ }^{\mathrm{c}}$ University of Paris 13, Sorbonne Paris Cité, CSPBAT Laboratory, UMR 7244, CNRS, Bobigny F-93000

${ }^{\mathrm{d}}$ Department of Chemical Sciences and Technology, University of Rome, Tor Vergata.

${ }^{\circledR}$ Istituto Nazionale di Biosistemi e Biostrutture (INBB)

* To whom correspondence should be addressed: massimiliano.aschi@ univaq.it

\section{Abstract}

A joint application of experimental and computational approaches has revealed the exceptionally high attitude of crabrolin, a 13-residue peptide with sequence FLPLILRKIVTAL-NH2, to adopt alpha-helix conformation not only in membranemimicking solvents but also in the presence of a not negligible amount of water. Our study shows that this propensity essentially resides in the intrinsic thermodynamic stability of alpha-helix conformation whose kinetic stability is drastically reduced in water solvent. Our analysis suggest that this is due to two effects enhanced by water; a more local effect consisting of the demolition of intra-peptide H-bonds, essential for the alpha-helix formation, and a bulk - electrostatic - effect favoring conformational states more polar than alpha-helix. 
Keywords: antimicrobial peptides - crabrolin - Nuclear Magnetic Resonance Circular Dichroism - Molecular Dynamics

\section{Introduction.}

Infectious microorganisms pervade the biosphere, deploying a potentially lethal threat to any life form. It is therefore not surprising that almost all multicellular organisms have evolved some type of defense system, either based on molecular and/or cellular components. The innate immune system provides such protection. In fact only recently (in evolutionary terms) vertebrates flanked, but did not replace it with the more sophisticated adaptive immunity, endowed with antigenic specificity and immunologic memory. Following immune recognition, inborn immune responses commonly include the release of antimicrobial peptides (AMPs), a large number of molecules differently active against bacteria, viruses, protozoa and fungi. After their initial discovery in insects and amphibians, hundreds of AMPs have then been identified and isolated from both prokaryotes and eukaryotes, either invertebrates or vertebrates, including humans [1-4]. With bacterial resistance to conventional antibiotics increasing at an alarming rate, and with the rising possibility of remaining practically unarmed against a growing number of noxious microbes, AMPs have received an ever-expanding dose of attention in the last three decades as potential constituents of a novel class of anti-infective therapeutic agents [5]. A large amount of experimental data has accumulated so far, indicating that AMPs act predominantly by disrupting the integrity of cell membranes through interaction with the phospholipids bilayer [6-10]. AMPs are essentially unstructured in the aqueous phase and fold upon contact with the membrane, adopting an amphiphilic structure. This conformation promotes the absorption of peptides onto the lipid bilayer and their subsequent integration into the membrane with expansion of the outer leaflet, which in turn leads to membrane thinning. The latter effect is not uniformly distributed over the entire bilayer area, but rather is concentrated in distinct domains [11]. Over a 
certain concentration threshold, peptides perturb membranes by forming transient pores via one of the various models proposed to account for this step, i.e. barrelstave, carpet-like, toroidal (or "wormhole") pore formation, detergent-type micellization, and induction of non-lamellar phases, leading to membrane permeabilization and either leakage of cell content and osmotic instability, and/or peptide diffusion to intracellular targets.

Previous work from our laboratories has reported Molecular Dynamics (MD) studies on a number of short natural peptides whom antimicrobial activity is strictly related to their amphypatic character, net positive charge and their propensity to adopt $\alpha$ helical conformation in hydrophobic solvent [12-16]. In this paper, we focused our interest on crabrolin, a 13 residues peptide whose sequence is FLPLILRKIVTALNH2 that has been found in the venom of European hornet Vespa Crabro, amidated at $\mathrm{C}$-terminus and with a net positive charge of $+2 /+3$ at neutral $\mathrm{pH}$, rich in hydrophobic aminoacids. This peptide was first discovered and studied by Argiolas e al. [17]. In particular, since for the majority of the above mentioned peptides the mode of folding appears a pivotal step for their interaction with the bacterial membrane, we describe the structural features of crabrolin in aqueous solution and in membrane-mimicking organic solvents, like TFE or HFA, by means of a joint application of experimental and computational approaches.

\section{Materials and methods}

\section{Experimental Details}

CD spectra were collected on a Jasco J600 Spectrometer (Jasco Inc., Easton, MD, USA) using a $0.1 \mathrm{~cm}$ path cell at $300 \mathrm{~K}$. Data were obtained from 198 to $260 \mathrm{~nm}$ at $0.2 \mathrm{~nm}$ interval, $20 \mathrm{~nm} / \mathrm{min}$ speed and averaging over four scans.

Spectra were collected by using a sample concentration of crabrolin of $66 \mu \mathrm{M}$ either in pure water or at different TFE/water $(\mathrm{v} / \mathrm{v})$ ratios, ranging from 100 to 0 , or in HFA/water solvent (50:50, v/v). Data are reported as mean residue ellipticity. 
NMR spectra were collected on a Bruker Avance $600 \mathrm{MHZ}$, equipped with a cryoprobe.

Crabrolin samples consisted of $1 \mathrm{mM}$ in either TFE/water solvent (50:50, v/v) or HFA/water solvent (50:50, v/v). For each sample TOCSY (50 ms and $90 \mathrm{~ms}$ spin lock times), NOESY (with mixing times of $150 \mathrm{~ms}$ and $300 \mathrm{~ms}$ for sample in TFE and mixing times of $50 \mathrm{~ms}$ and $150 \mathrm{~ms}$ for the sample in HFA) and natural abundance $\mathrm{C}^{13}$-HSQC were collected at a temperature of $298 \mathrm{~K}$. 800 increments were collected for the NOESY and TOCSY data, 256 increments for the natural abundance HSQC spectrum.

Data were processed with NMRPipe [18], and spectral analysis was performed with Sparky [19] by using the NMRFAM version [20].

Structure calculations were performed by using CYANA, version 3.97 [21].

For the sample in TFE/water (50:50, v/v), 273 useful symmetrical NOEs from the 150 ms NOESY experiment were used that were translated in 112 final peaks in the last CYANA cycle of calculation (97 short-range, $|\mathrm{i}-\mathrm{j}|<=1 ; 15$ medium-range, $1<\mid \mathrm{i}-$ $\mathrm{j} \mid<5$.

The final and initial target function values were 0.59 and 80.92, respectively.

For the sample in HFA/water (50:50, v/v), 208 useful symmetrical NOEs from the 50 ms NOESY experiment were used that were translated in 106 final peaks in the last CYANA cycle of calculation (91 short-range, $|\mathrm{i}-\mathrm{j}|<=1 ; 15$ medium-range, $1<|\mathrm{i}-\mathrm{j}|<5$.

The final and initial target function values were 0.10 and 36.25 , respectively.

Molecular graphics and analysis were performed with the UCSF Chimera package [22] or with MOLMOL software package [23].

\section{Computational Details}

MD simulations were performed utilizing the Gromacs package [24]. Crabrolin was initially put in the fully extended configuration in a cubic box filled with 3360 molecules described by the single point charge (SPC) model [25] for the simulation in water. For the simulations in TFE we utilized 820 molecules described by an all- 
atom force field contained in the Gromacs package with the charges calculated by Van Buuren and Berendsen [26]. A third simulation was also carried out using a mixed solvent consisting of water and TFE approximately at the same molar ratio. This latter solvent is hereafter termed as 50:50. After an energy minimization, the whole system was slowly heated up to $300 \mathrm{~K}$ using short (100.0 ps) MD runs and the isothermal/isochoric ensemble (NVT) was adopted in all the simulations making use of the velocity rescaling procedure [27] and time of $2.0 \mathrm{fs}$ was used as integration step. Before the production of the simulations of $300 \mathrm{~ns}$ we adjusted the dimensions of all the boxes to reproduce the correct density at the temperature and pressure of the simulation using the following protocol: first, the box filled with the pure solvent (either water or TFE) was simulated at the typical density under the pressure of 1.0 bar; this simulation, carried out in the NVT ensemble, produces an average pressure termed as $\bar{P}$. Then the crabrolin was inserted in the box and the dimension of the whole system was adjusted to reproduce the same $\bar{P}$ previously registered in pure solvent simulation. Crabrolin was described using the OPLS force field [28], the LINCS algorithm was adopted to constrain all bond lengths [29], and the long range electrostatics were computed by Particle Mesh Ewald method [30] with 34 wave vectors in each dimension and a $4^{\text {th }}$ order cubic interpolation. Much of the collective analysis used in this work is based on Essential Dynamics (ED). Details of ED are reported in the literature [31] and here we only report some specific features for the benefit of not-expert readers. MD trajectories of peptides and proteins, and in general of systems formed by a large number of atoms, are very difficult to interpret because of the enormous number of degrees of freedom. In other words any attempt of extracting from MD trajectory the conformational features of a large molecule based on the personal intuition is likely to produce arbitrary and incomplete results. ED, and also other approaches based on cluster-analysis, may provide unbiased. The first step of ED is the construction of the positional covariance matrix of the peptide whose roto-translational coordinates have been preventively removed

$$
\tilde{C}=\left\langle(x-\langle x\rangle) \cdot(x-\langle x\rangle)^{T}\right\rangle
$$


In matrix (1) $\boldsymbol{x}$ represents the atomic coordinates and $\langle\boldsymbol{x}\rangle$ the corresponding average along the simulation. Matrix (1) is then diagonalized producing a set of eigenvectors $\left(\boldsymbol{\mu}_{i}\right)$ and associated eigenvalues. The eigenvectors represent new (internal) coordinates, alternative to the Cartesian coordinates, along which the peptide fluctuations occur and whose extent is represented by the associated eigenvalues. It follows that the trace of the matrix (1) represents a direct measure of the extent of the whole fluctuation of the peptide. The eigenvectors of matrix (1) may in principle greatly simplify the conformational analysis. As a matter of fact, as demonstrated in the literature [31], it is possible to separate the whole peptide conformational space into two subspaces: (i) a space formed by all the eigenvectors showing low eigenvalues and hence representing conformational coordinates along which the peptide undergoes quasi-harmonic (quasi-constrained) motions; (ii) a second space, termed as Essential Subspace, formed by $\mathrm{M}$ eigenvectors $\left(\boldsymbol{\mu}_{1}, \boldsymbol{\mu}_{2}, \ldots \boldsymbol{\mu}_{M}\right)$ with the highest eigenvalues, i.e. the conformational fluctuations. This subset of eigenvectors can be used to reconstruct the peptide conformational pattern. This can be accomplished by projecting the coordinated of the peptide atoms onto the essential space through equation (2)

$p_{i}(t)=\boldsymbol{\mu}_{i} \cdot(\boldsymbol{x}(t)-\langle\boldsymbol{x}\rangle) \quad(i=1, M)$

This produces the displacement along the Essential Subspace. If such a Subspace is characterized by a reduced number of eigenvectors (typically 1 or 2 for small peptides), the projection produces a number of spots, termed as conformational basins, into the Essential Subspace hence providing a very efficient and relatively easy procedure for identifying the peptide conformations. It follows that if we simulate (see below) the same peptide in different conditions (different trajectories), drastic differences in the conformational repertoire can be quantitatively obtained by comparing the corresponding essential eigenvectors. At the same time a more direct, and less quantitative, estimation of the level of similarity of conformational patterns can be also performed by comparing the corresponding projections (spots) onto a 
common Essential Subspace, e.g. trajectories (a) and (b) both projected on the Essential Subspace of (a). In this case the absence of any overlap, or the presence of a scarce overlap, between the spots indicates that the peptide is spanning a different conformational space in the two trajectories (see Results section)

\section{Results.}

\section{CD experiments.}

$\mathrm{CD}$ spectra in water and at increasing TFE/water ratio are reported in Figure 1. The data show that crabrolin is essentially random coil in water and assumes a helical conformation even at low TFE/water (20:80, v/v). Only slight changes are observed at higher TFE/water ratio, suggesting that the peptide has a strong helical propensity. Helical content was evaluated to be $74 \%$, based on the method proposed by Luo and Baldwin [32] and by using a $\mathrm{k}$ value of 4 . 


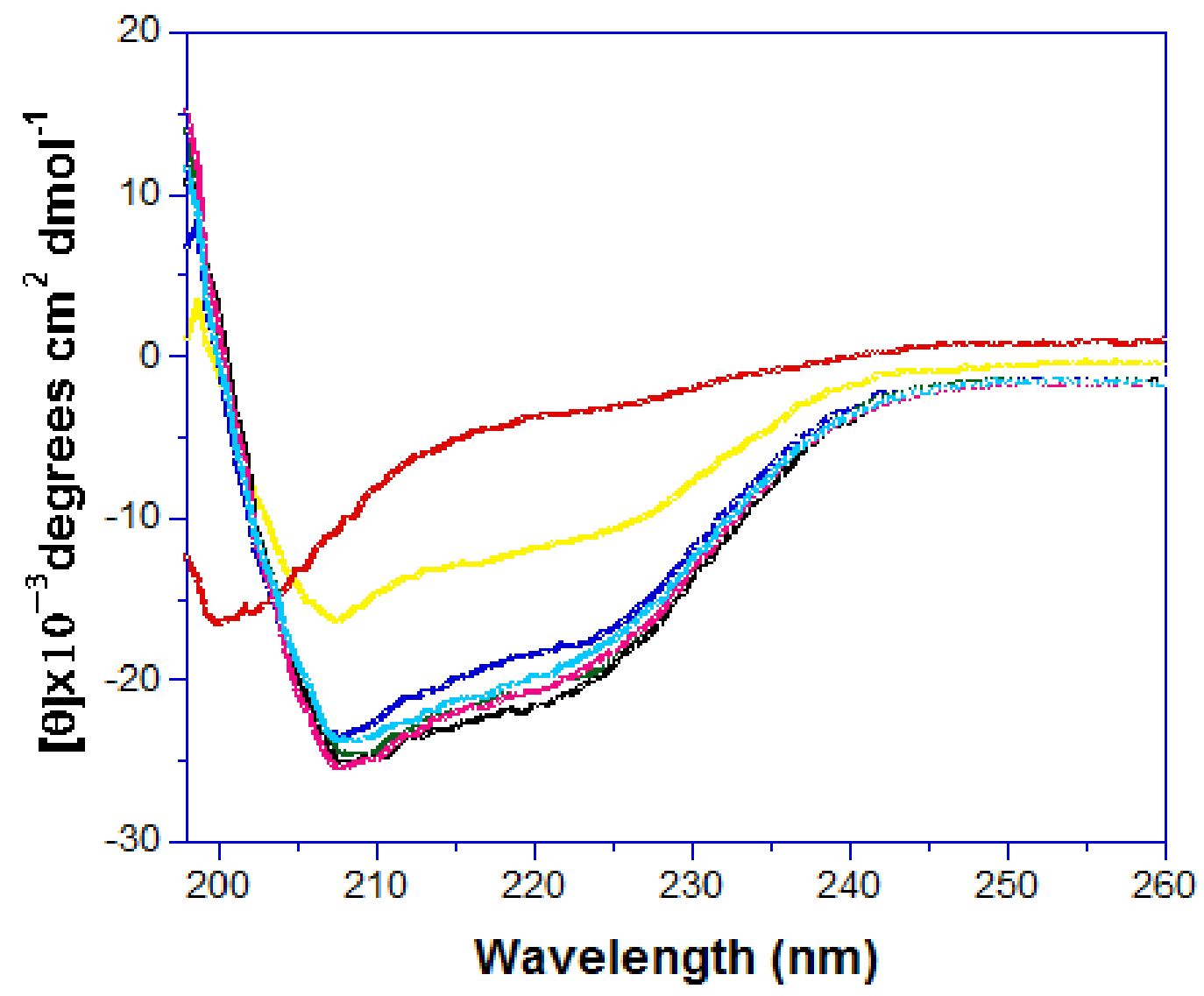

Figure 1.

Circular Dichroism spectra of crabrolin. The spectra were collected in water (red line), and at increasing TFE/water (v/v) ratio: 20\% (blu line); 40\% (green line); 60\% (black line); 80\% (magenta line), and 100\% (cyan), respectively. Spectrum in HFA/water (50:50, v/v) is in yellow. 


\section{NMR experiments.}

In order to confirm the previous suggestions provided by $\mathrm{CD}$ spectra we performed NMR spectra in the mixed TFE/water solvent $(50: 50, \mathrm{v} / \mathrm{v})$ to ascertain the strong folding propensity at a relatively high water concentration.

Similarly, a different solvent, composed of HFA/water (50:50, v/v) was also used since it has been reported that this system is a valid alternative to TFE [33].

Comparison in two different environments may help indeed to elucidate different folding propensities of the peptide.

NMR spectra (see Supplementary Information) were analyzed using the classical Wuthrich sequential assignment approach [34]. In order to assign the side chains of the peptide, a natural abundance ${ }^{13} \mathrm{C}-\mathrm{HSQC}$ was also collected. Assignment of peptide resonances in both systems is reported in the Supplementary Information.

Structure calculation was performed by using CYANA [21] and the 20 structures with the lowest target function were selected (see Supplementary Information).

In the presence of TFE/water solvent $(50: 50, \mathrm{v} / \mathrm{v})$ the final target function value for the selected structures is 0.59 . The average backbone RMSD to mean is $0.09+/-0.09$ $\AA$ and the average heavy atom RMSD to mean is $0.54+/-0.08 \AA$ indicating that the structures are convergent. $91 \%$ of the residues are located in the allowed regions of the Ramachandran plot. No NOE violations were observed.

The structures obtained in water/TFE show the presence of alpha-helix between residues 3 and 11 as evaluated by measuring the phi/psi angles

A superposition of the 20 structures is reported in Figure 2.

In the presence of HFA/water solvent $(50: 50, v / v)$ the final target function for the 20 selected structures is 0.1 . The average backbone RMSD to mean is $0.25+/-0.03 \AA$ and the average heavy atom RMSD to mean is $0.66+/-0.06 .91 \%$ of the residues are located in the allowed regions of the Ramachandran plot. No NOE violations were observed.

Also in this case, the structures show the presence of alpha-helix between residues 3 and 11 and the final structures are very similar to the ones obtained in TFE/water. 
Inspection of the structures shows that the charged residues (arginine and lysine) are located on the same side, suggesting that this part of the molecule could interact with the membrane.

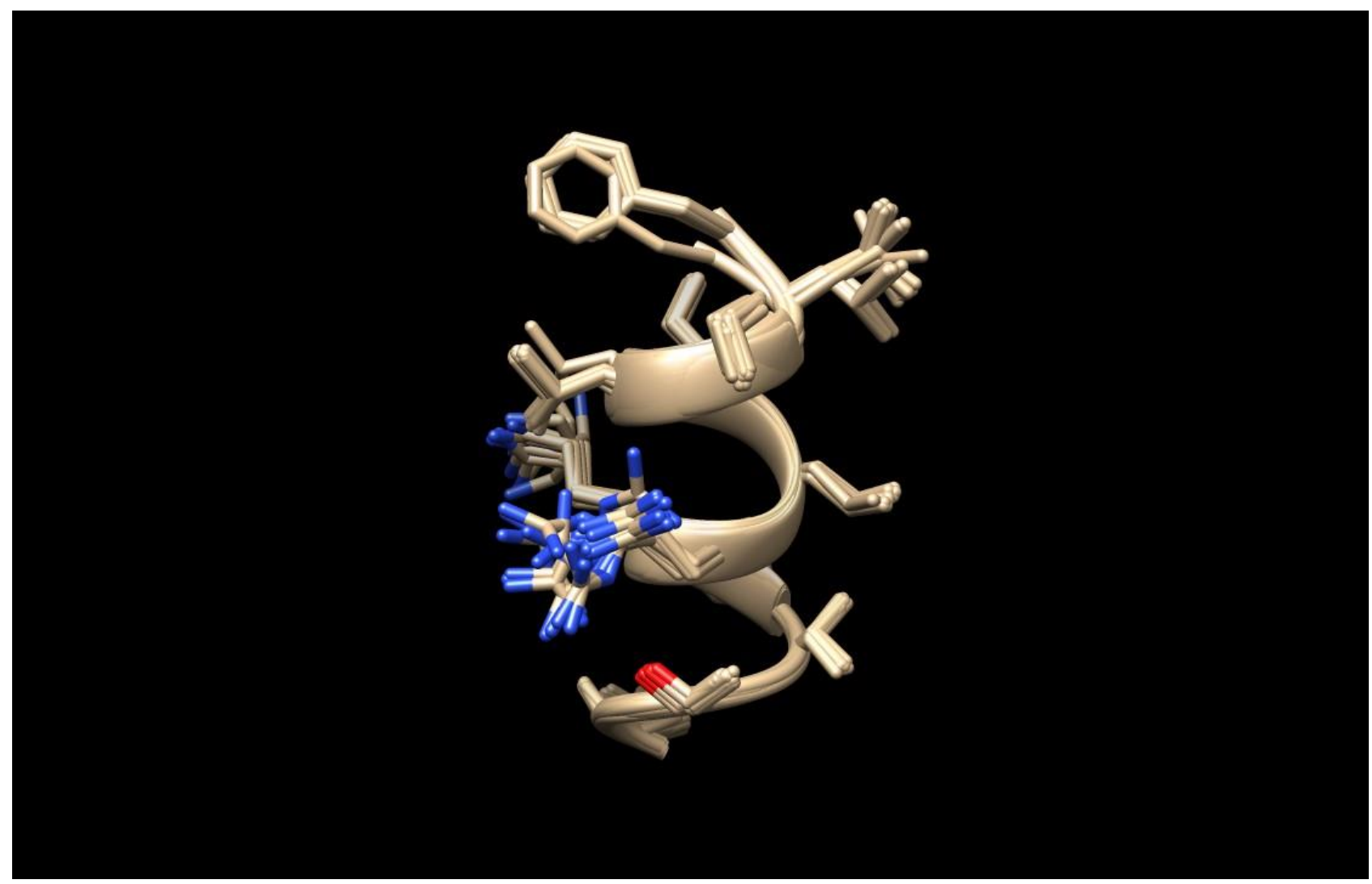

\section{Figure 2.}

NMR solution structure of Crabrolin in a mixed TFE/water solvent (50:50, v/v). Superposition of the 20 lowest structures obtained after torsion angle dynamics. The backbone is depicted as a ribbon. The structure was obtained with Chimera [21]

\section{Molecular Dynamics simulations}

The high propensity of crabrolin to fold in alpha-helix, and in general the conformational analysis, was checked using MD simulations in the different environments.

Internal flexibility of the crabrolin, evaluated by the Root Mean Square Fluctuation (RMSF) reported in Figure 3, reveals as expected, a markedly different behavior 
depending on the solvent used. In water crabrolin shows much higher fluctuation pattern suggesting a conformational repertoire wider than the one in TFE.

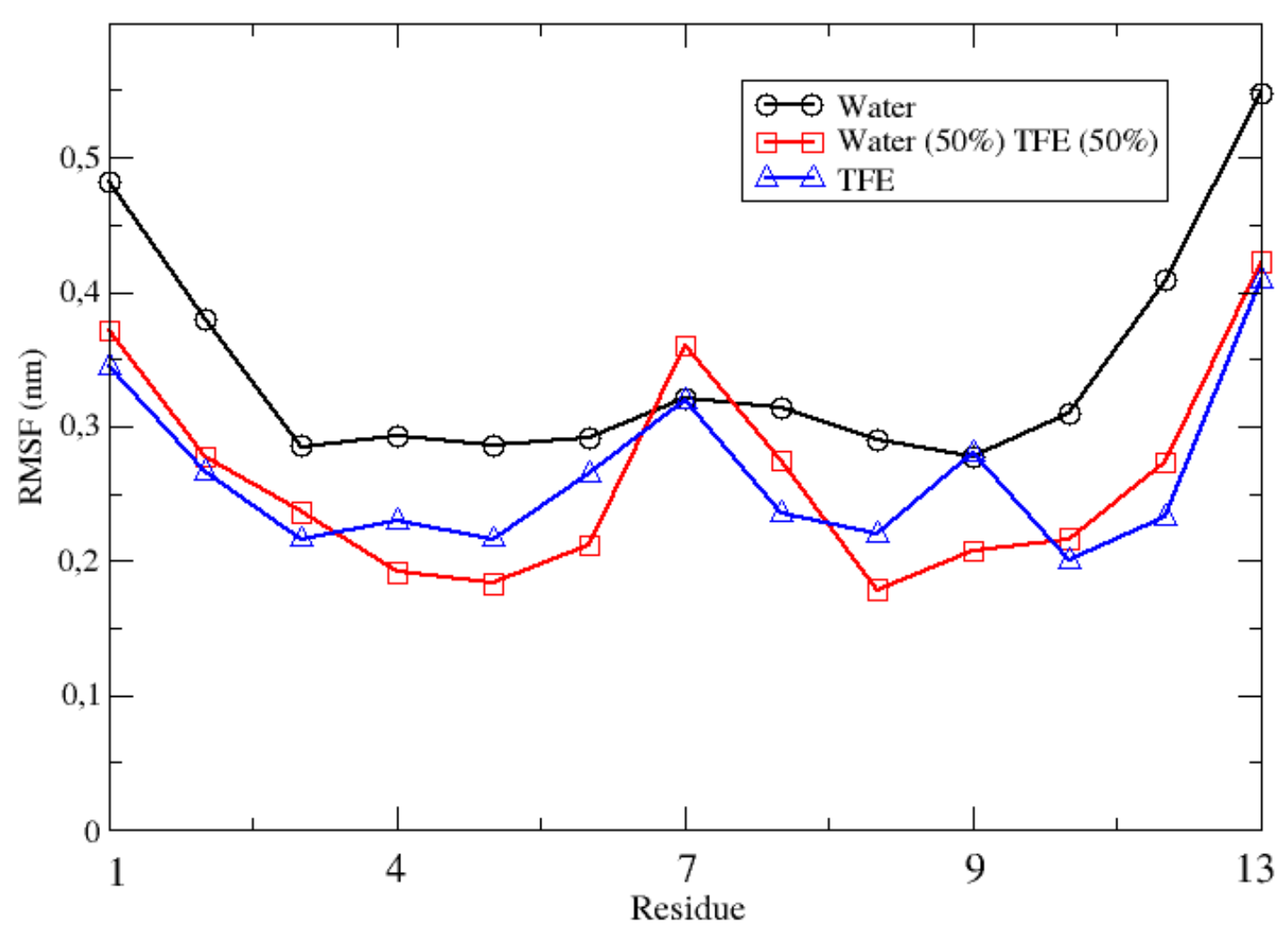

Figure 3. C-alpha Root Mean Square Fluctuation of Crabrolin in the different investigated solvents

Interestingly and qualitatively in agreement with the previous spectral data, crabrolin fluctuation in the 50:50 solvent is markedly more akin the one in TFE.

A more detailed characterization of the structural-conformational features was then accomplished by using ED analysis as described in the Computational Details section.

For each simulation we evaluated the crabrolin C-alpha covariance matrix (1) whose diagonalization produces a number of eigenvectors. The spectrum of the corresponding eigenvectors is reported in Figure 4 for the three simulations. 


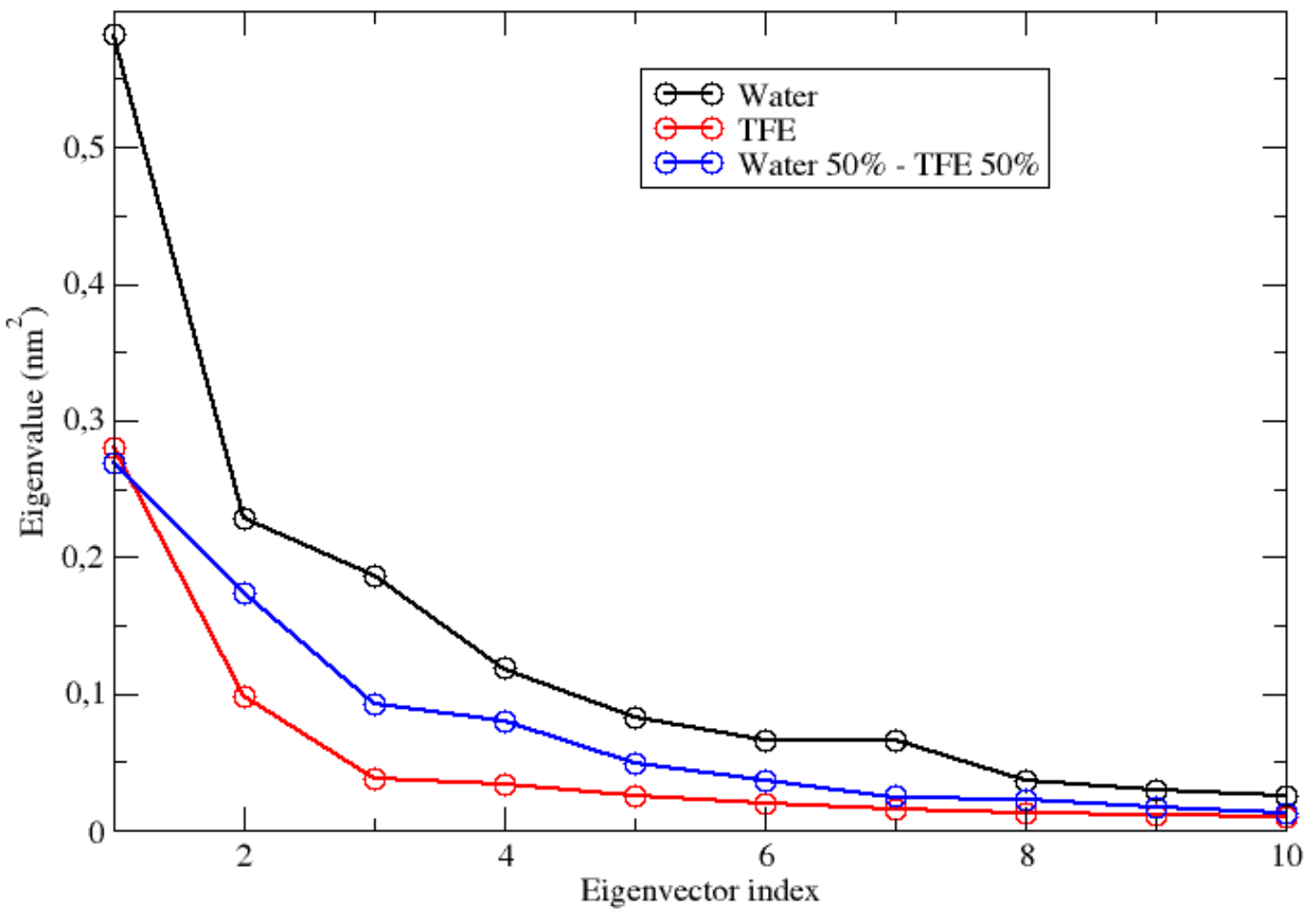

Figure 4. Spectrum of the eigenvalues of covariance matrix of crabrolin in different solvents (Black : water; Red: TFE; Blue: 50:50 solvent). Note that for the sake of clarity only the first 10 eigenvalues are reported.

Results essentially parallel the features emerged by RMSF analysis (Figure 4): the largest fluctuation is observed in water and, at the same time, TFE and 50:50 solvent are characterized by a similar patterns.

Moreover the evaluation of the overlap between the covariance matrices (1), resulting equal to 0.48 (between TFE and Water) and equal to 0.70 (between TFE and 50:50 solvent) confirm the differences and similarities already suggested by the fluctuation analysis as explained in the Computational Details section.

It is also important to note that according to Figure 4, and not surprisingly when small peptides are concerned [35], the whole fluctuation is almost completely (more 
than 60\%) confined within the first two eigenvectors, the Essential Subspace, which then can be used to project the trajectories (see equation (2) in the Computational Details section).

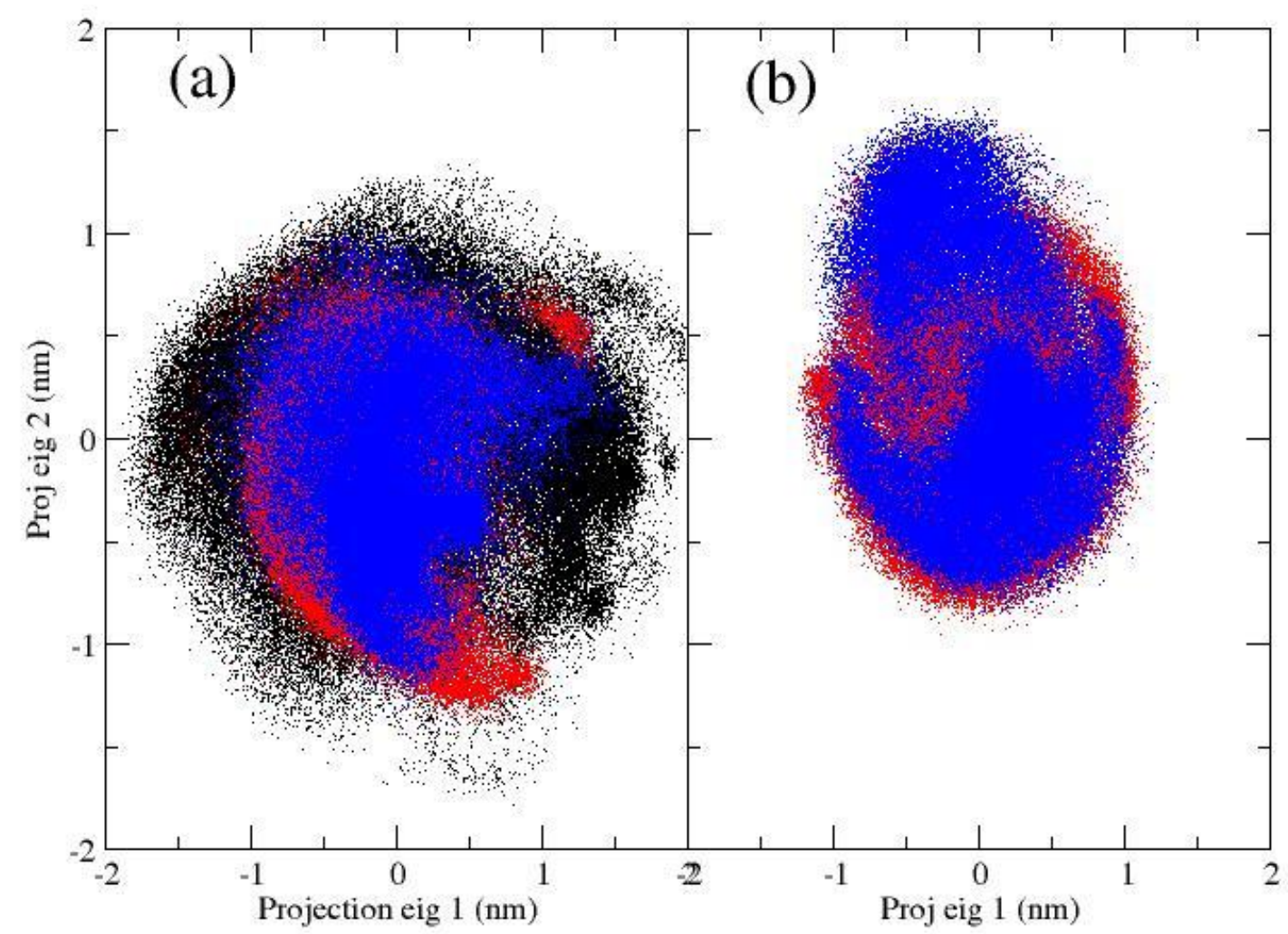

Figure 5. Projection of the trajectory in water (black), TFE (red) and 50:50 solvent (blue) onto the water essential plane (panel a) and TFE essential plane (panel b)

Further comparison between the conformational patterns in the different solvents has been accomplished, as described in the Computational Details section, by projecting the different trajectories onto common Essential Subspaces. In the panel (a) of the Figure 5 we show the result of the projection of all the three simulations onto the 
Essential Subspace in water whereas, in the panel (b), we report the projection of the TFE and 50:50 trajectories onto the same Essential Subspace in TFE.

From the Figures it is evident that, at least within our simulation setup, the whole conformational space spanned by crabrolin in TFE and 50:50 solvent represents a fraction of the conformational space also spanned in water.

In other words we can expect that all the conformations sampled by crabrolin in TFE and 50:50 are also found in water, but not vice versa.

At the same time the high resemblance between the conformational space of crabrolin in TFE and 50:50, previously evaluated by the covariance matrix overlap, is confirmed by the overlap of the spots blue and red in the Figure 5(b).

More quantitative structural analysis has been performed by calculating the (Helmholtz) free energy profile as a function of the essential eigenvectors using the usual relation:

$$
A(q)=-R T \ln \frac{\rho(q)}{\rho\left(q_{\text {ref }}\right)}
$$

with $q$ and $q_{\text {ref }}$ indicating, respectively, a generic position onto the essential plane (i.e. a conformational basin) and a reference, arbitrarily selected, position (i.e. the reference conformational basin) of the peptide coordinates along the essential plane. $\rho(q)$ and $\rho\left(q_{r e f}\right)$ are the probability densities representing the number of times the projected trajectory falls onto the given conformational basin divided by the total number of frames.

Note that differently from the Figure 5, in this analysis each trajectory has been projected onto its own essential plane.

Note also that given the high similarity between TFE and 50:50 trajectories, we show the results only for water and TFE reported in Figure 6 and Figure 7, respectively. In the same Figures we have also highlighted the most relevant crabrolin conformations schematically depicted in the upper part of the Figures. 


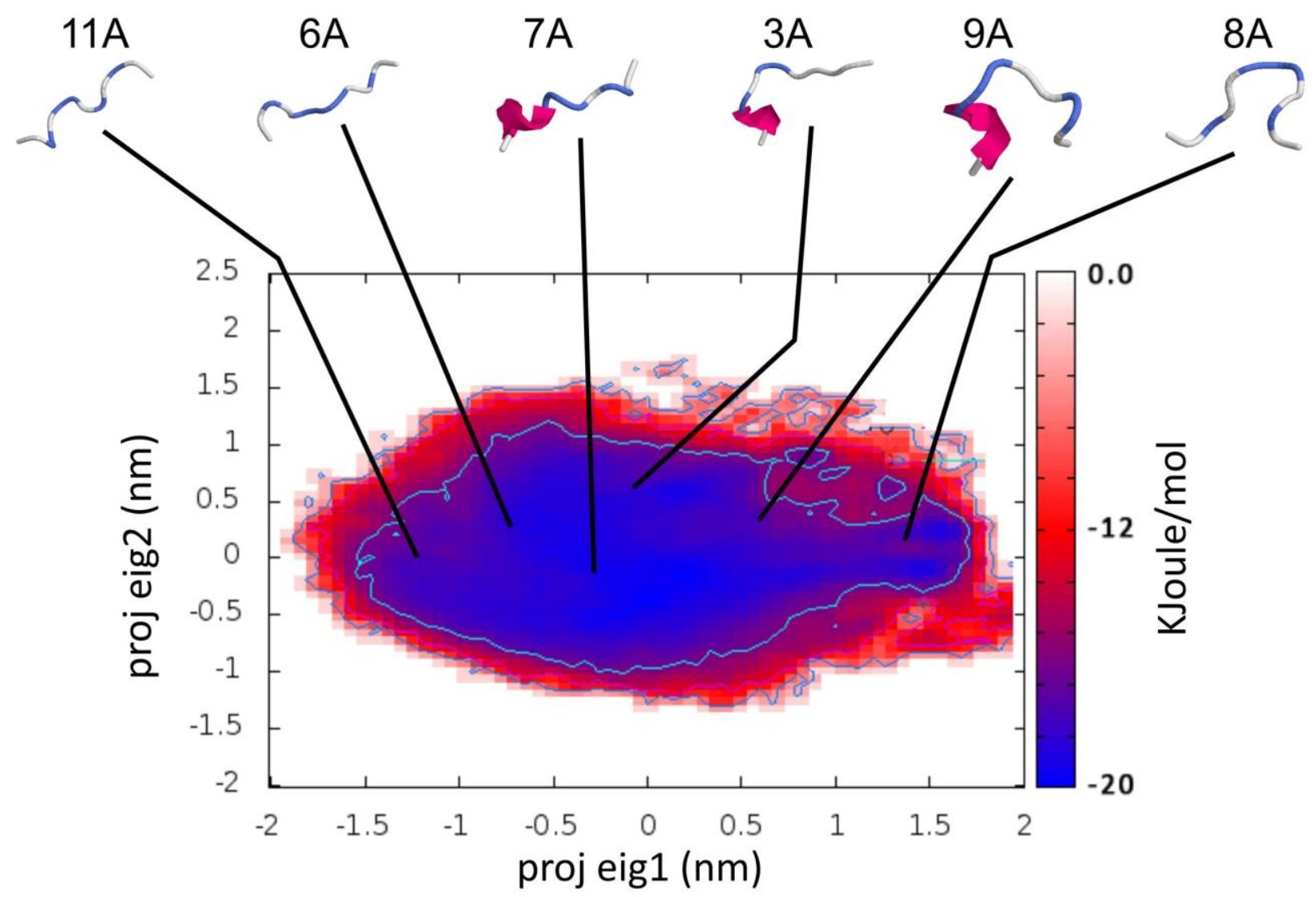

Figure 6. $300 \mathrm{~K}$ free energy profile (in kJoule/mol) evaluated onto the essential plane (see Figure 6 panel (a)) of aqueous crabrolin.

From Figure 6 it is evident that crabrolin in water is characterized by a very flat free energy surface, in line with the already remarked high RMSF, thus indicating the presence of a high number of conformations in rapid interconversion not in disagreement with the unfolded state emerged from the $\mathrm{CD}$ spectra.

From the inspection of the representative conformations, reported in the upper side of the same Figure, the central region of the whole conformational space turns out to be the basin resembling the alpha-helix conformational state (termed as alpha-helix basin). This finding points out an intrinsic tendency of aqueous crabrolin to fold in alpha-helix which represents a thermodynamically accessible, but kinetically unstable, state in water. 
It is worth to note, from comparison between the Figure 5 panel (a) and Figure 6, that the conformational space accessed by crabrolin both in TFE and in 50:50 solvent, perfectly fits the alpha-helix basin hence suggesting a high stability of alpha-helix conformation in these solvents.

This hypothesis is indeed confirmed from the Free energy landscape of the Figure 7 where we effectively observe that the crabrolin conformational space in TFE is characterized by a reduced area (lower RMSF) and deeper free energy minima, i.e. kinetically more stable, with respect to the situation in water.

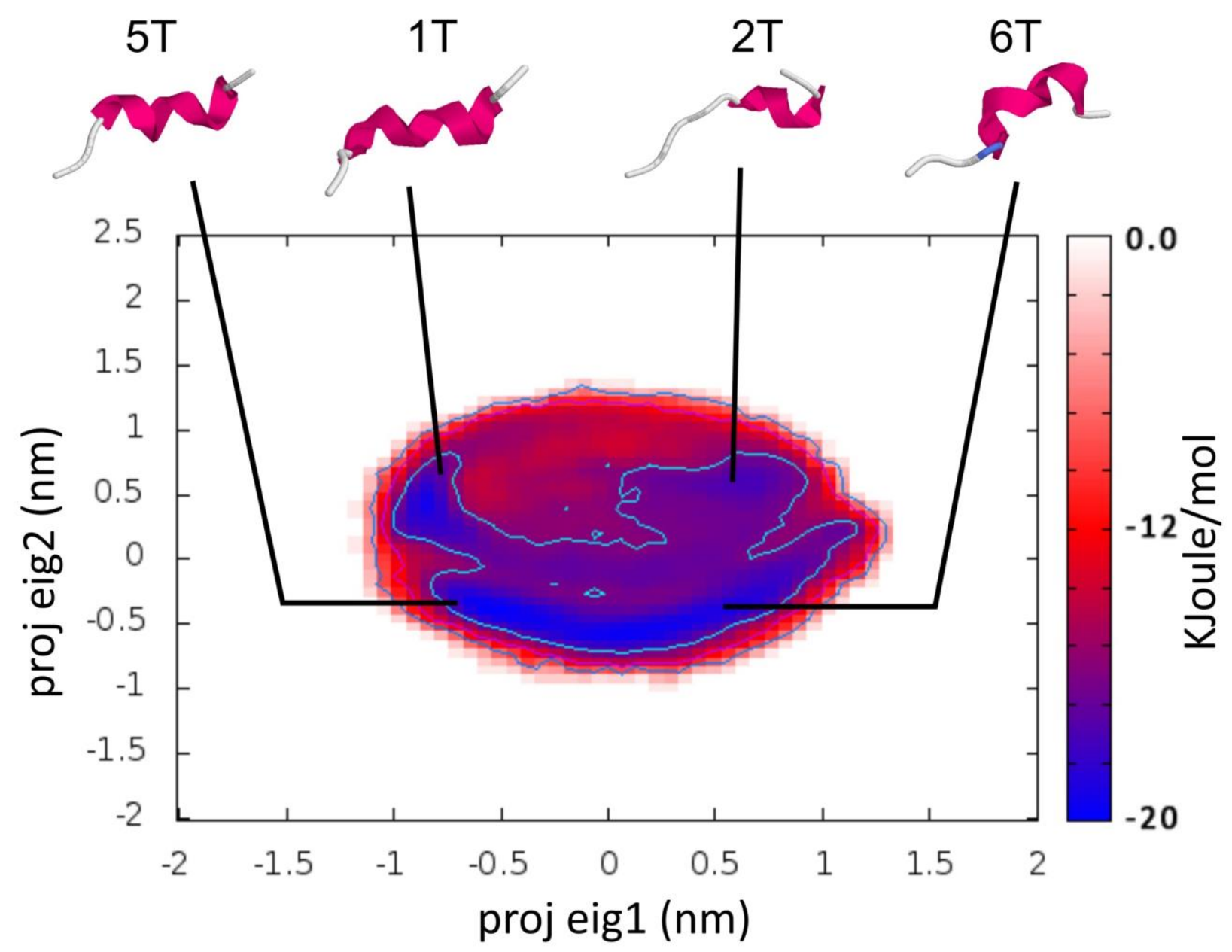

Figure 7. 300K free energy profile (in kJoule/mol) evaluated onto the essential plane (see Figure 6 panel (b)) of crabrolin in TFE. 
The emerged picture is in satisfactory agreement with the experimental data showing the presence of a stable (thermodynamically accessible but also kinetically stable) alpha-helix conformation.

For further rationalizing the previously shown behavior we have finally analyzed some typical observables as provided by MD simulations. The results of these observables have been then correlated with the crabrolin conformations by calculating the average values of the observables in each of the free-energy basins of Figures 6 and 7. In particular we have extracted: (a) the number of intra-peptide hydrogen bonds (using the default definition present in Gromacs concerning donor acceptor distance and $\mathrm{H}-\mathrm{O}-\mathrm{H}$ angle); (b) the norm of the crabrolin electric dipole moment. i.e. the peptide polarity; (c) the hydrophobic and hydrophilic solventaccessible surfaces as provided by standard criteria [36]; (d) an estimation of the peptide volume and shape. This latter quantity has been accomplished by using a recently proposed method [37] based on elementary mechanics and here only briefly outlined.

For each simulation, and at each frame, we have constructed the $3 \times 3$ covariance matrix $\widetilde{M}$

$\widetilde{M}=\frac{1}{N} \sum_{i=1}^{N}\left(\boldsymbol{x}_{\boldsymbol{i}}-\langle\boldsymbol{x}\rangle\right) \cdot\left(\boldsymbol{x}_{\boldsymbol{i}}-\langle\boldsymbol{x}\rangle\right)^{T}$

where $\mathrm{N}$ is the total number of atoms of the protein and $\langle\boldsymbol{x}\rangle$ is a vector whose components are the average positions ( $\mathrm{x}, \mathrm{y}$ or $\mathrm{z}$ ) of all the atoms at the given frame. Diagonalization of matrix (3) produces three eigenvectors representing the axis of an instantaneous ellipsoid best approximating the shape of the peptide. The values of the above axes $\left(a_{i}\right.$, with $\left.\mathrm{i}=1,2,3\right)$ are hence given by $a_{i}=2 \sqrt{ } l_{i}$ where $l_{1}, l_{2}$ and $l_{3}$ are the associated eigenvalues of matrix (2). The crabrolin volume, at each particular frame, has been then approximated by the volume of the associated ellipsoid. The calculation has been carried out twice: one for the whole peptide and one only for the backbone for estimating the effect of the side-chains. 
WATER

(a)

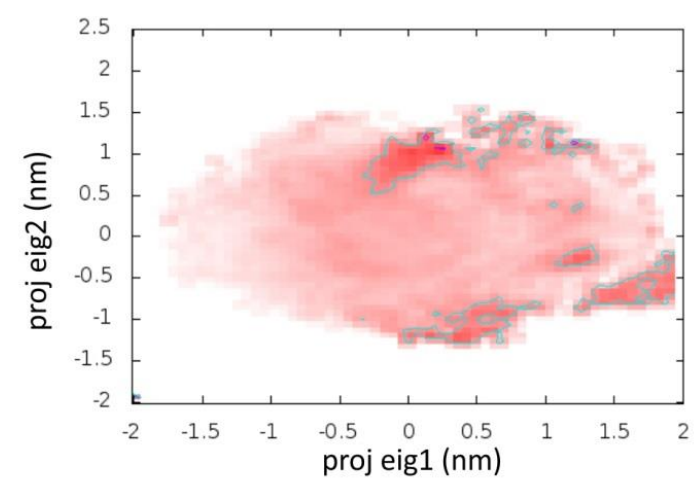

(b)

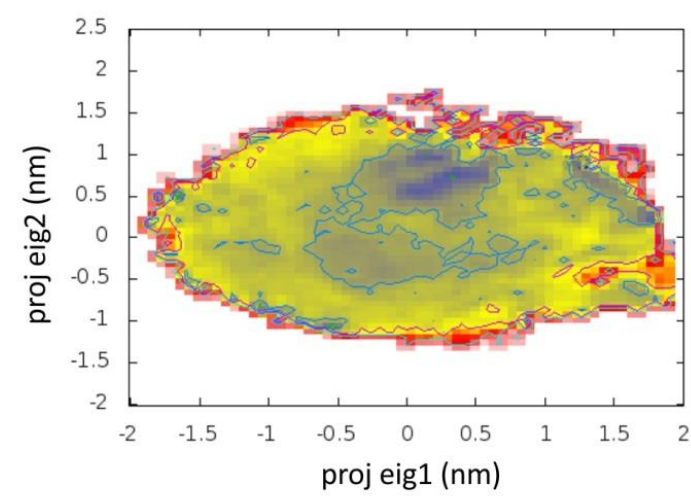

TFE
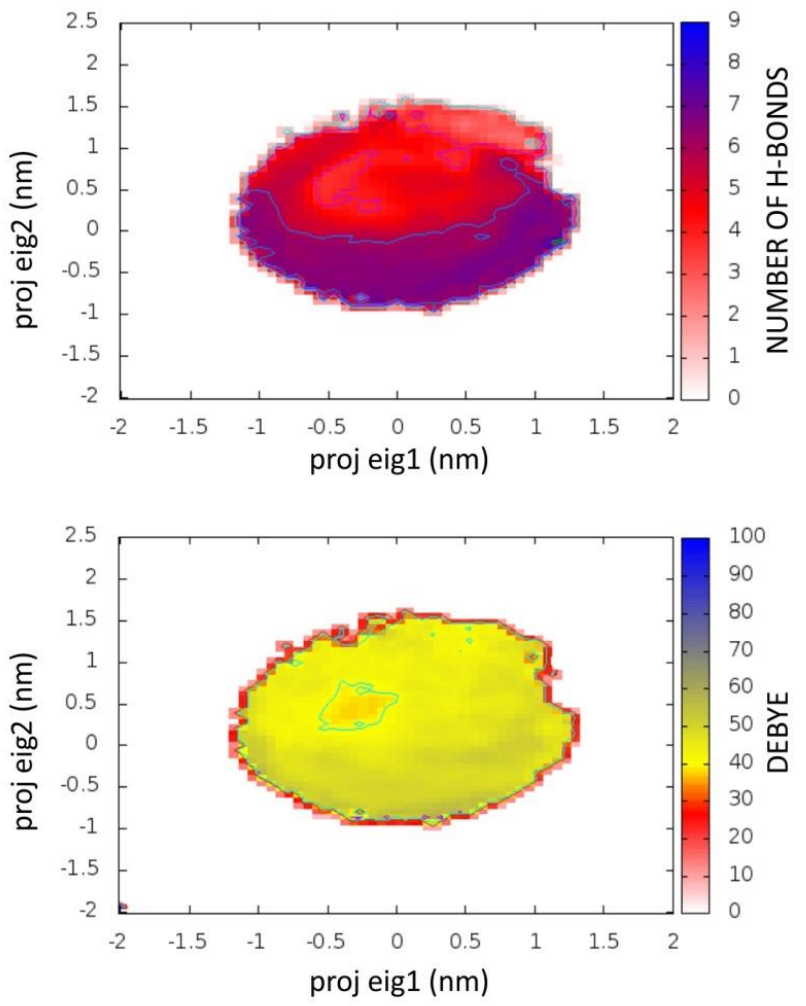

Figure 8 Panel (a) average number of intra-peptide hydrogen bonds in each conformational basin. (b) average norm of crabrolin electric dipole moment (in Debye) in each conformational basin. See Figures 6 and 7 for comparison. 
WATER

(a)

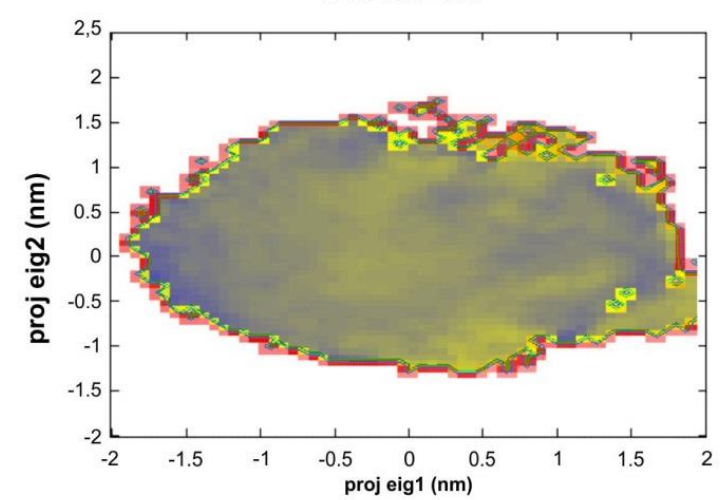

(b)

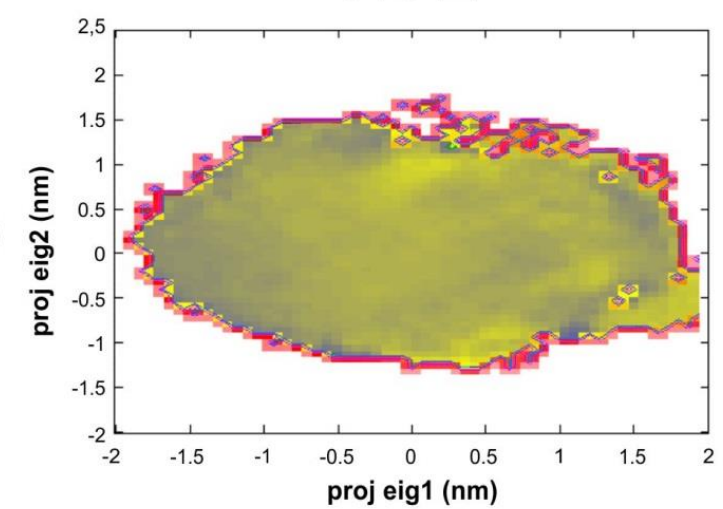

TFE
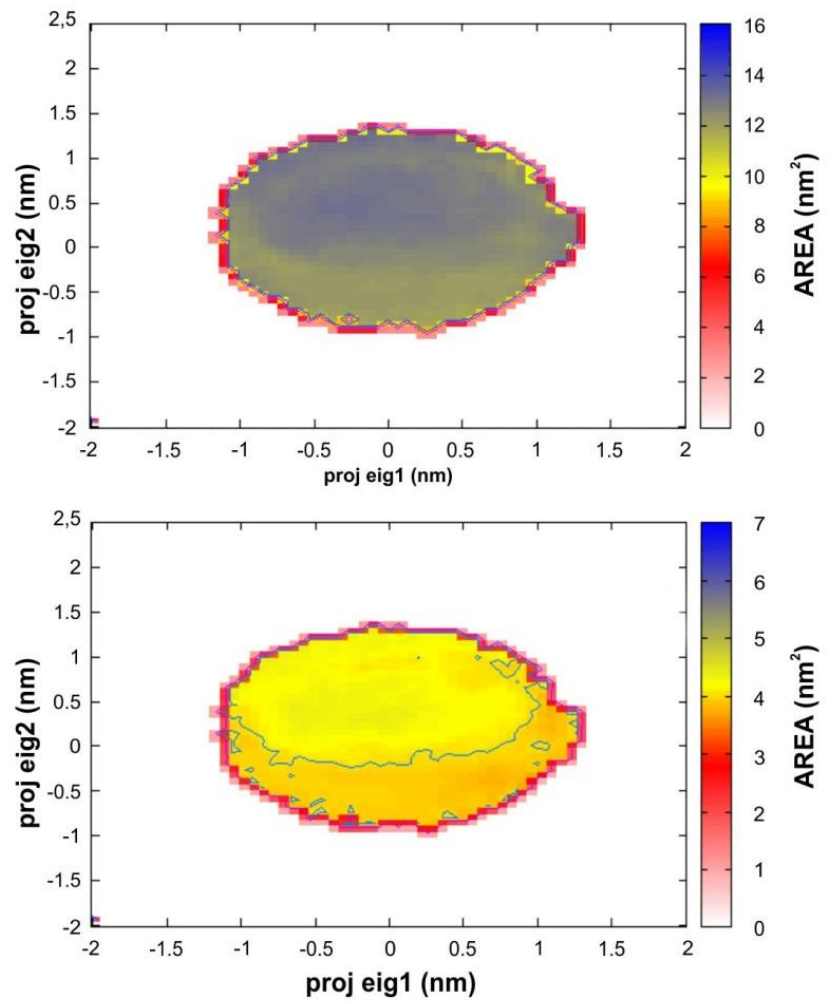

Figure 9. Panel (a) average value of the hydrophilic surface $\left(\mathrm{nm}^{2}\right)$ in each conformational basin. (b) average value of hydrophobic surface $\left(\mathrm{nm}^{2}\right)$ in each conformational basin. See Figures 6 and 7 for comparison. 
WATER

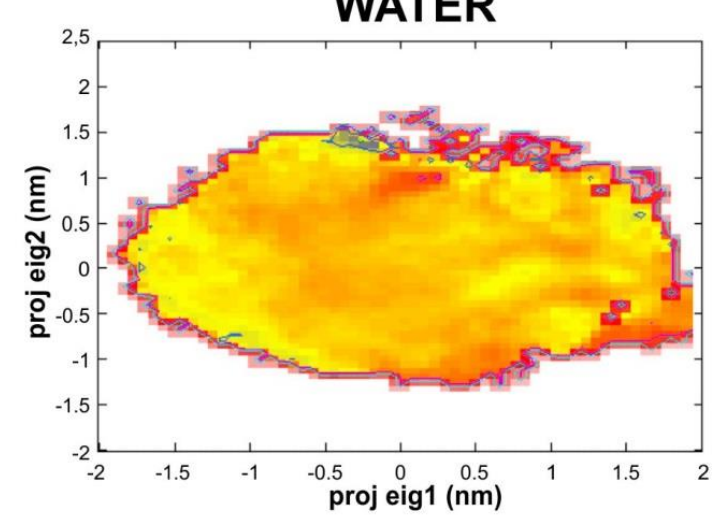

(a)

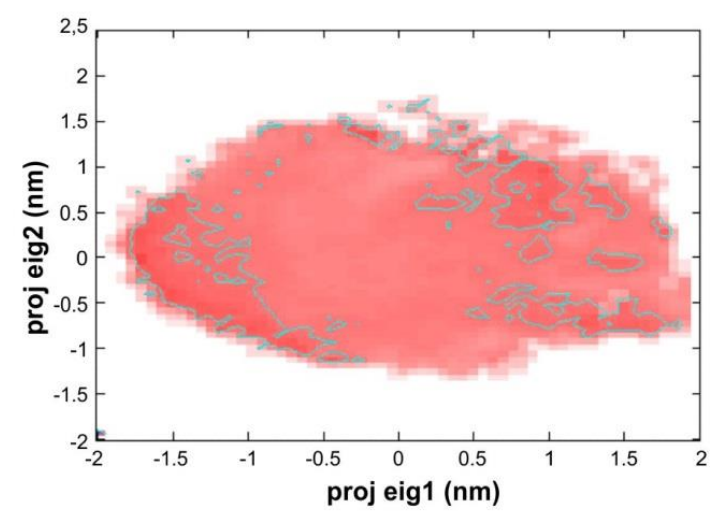

TFE
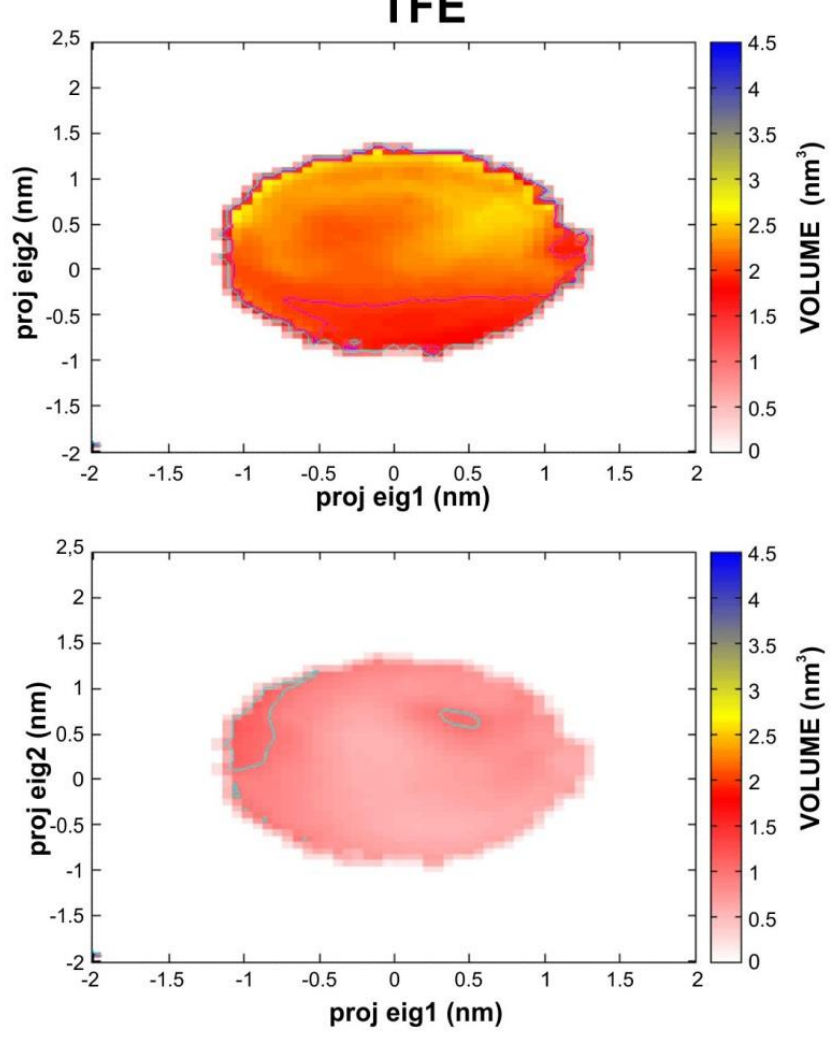

Figure 10. Average value of the crabrolin volume (from the ellipsoid obtained from matrix (3)). In the panel (a) the whole peptide is considered. In panel (b) only the backbone is taken into account

The results collected in the Figures 8, 9 and 10 can be summarized in a few key points:

- A dramatic reduction of intra-peptide H-bonds is observed by comparing crabrolin in water (Figure 8 panel a) and in TFE (same Figure, panel b). This effect is obviously due to the presence of competing H-bonds between solute and solvent much higher in water. At the same time comparison between Figures 6 and 7 with Figure 8(b) suggests that the stability of the crabrolin conformational states in water seems to parallel the polarity of the peptide which is slightly but significantly enhanced (from 10 to 20 Debye) in correspondence of the most stable (negative relative free energy) conformations characterized by an incipient hairpin-like conformation (structure 3A of Figure 6). Deeper inspections indicate that this is mainly 
related to the participation of the polar side chains which in water arrange in a more solvent-exposed fashion increasing the peptide hydrophilic surface (Figure 9 panel a) and also producing a sharp enhancement of the whole peptide volume (Figure 10 panel a) while maintaining the backbone volume (Figure 10 panel b) comparable with that in TFE.

- Concerning crabrolin in TFE we observe that the most stable conformations (if compared to the less stable ones), corresponding to well-defined alpha-helix structures, are characterized by a more stable network of intra-peptide $\mathrm{H}$ bonds, a markedly more compact structure, i.e. lower volume (Figure 10), with also a slight but significant decrease of the both the hydrophilic (Figure 9 panel a) and hydrophobic (Figure 9 panel b) areas. Worth of remark is also the fact that pure alpha-helix conformational states show a slightly reduced dipolemoment with respect the incipient alpha-helix folded states (e. g, structure 7A of Figure 6) adopted by crabrolin in water.

\section{Discussion.}

We have analyzed the behavior of crabrolin, an antimicrobic peptide, in different solvents using a combination of experimental and computational approaches. Experimental data consisted of analysis based either on CD or NMR spectroscopy. CD spectra show a very high propensity of crabrolin to fold in alpha-helix in organic solvents and a lack of folded structure in water. CD spectrum of crabrolin in TFE was already reported by Krishnakumari et al. [38]. The profile showed a quite similar pattern but a more pronounced minimum at $208 \mathrm{~nm}$, compared to that reached at 222 $\mathrm{nm}$, was found with respect to our values. The reason for this discrepancy could be 
probably due to an equilibrium between turn structures and alpha helix domains. In addition, in our experiments spectra were collected by using crabrolin samples dissolved either in pure water or in increasing concentrations of TFE while in Krishnakumari's spectra, crabrolin was dissolved in 5 mM HEPES, pH 7.4.

The presence of alpha-helix has been better characterized by NMR spectroscopy. In TFE/water (50:50, v/v) and in HFA/water (50:50, v/v), the NOESY spectra show the typical cross peaks appearance of helical structure.

From the analysis of such spectra a 3D structure has been obtained using torsion angle dynamics as implemented in the CYANA software.

In both case the presence of alpha-helix is clearly detected between residue 3 and 11 that represent the core of the peptide.

Inspection of the structures shows that the charged residues (arginine and lysine) are located on the same side of the helix, suggesting that this part of the molecule could interact with the membrane if its mode of action is to lean against to the membrane.

Additional information derived from MD simulations confirms the high tendency of crabrolin to fold in alpha-helix resulted as a stable conformational state thermodynamically favored both in water and in TFE.

The differences between the two conditions is the kinetic stability of apha-helix rather scarce in water essentially because of the disturbing effect of the solvent molecules both at local level - disruption of the intra-peptide H-bonds network - and both as a bulk effect leading the crabrolin to more polar favoring conformations more polar than TFE.

On the other hand TFE molecules, because of their dimensions and local polarity, are much less harmful for the H-bond network and, at the same time, are less able to drive crabrolin toward more polar conformational states different from alpha-helix. The presence of a reduced number of TFE molecules is evidently sufficient to enhance the kinetic stability of alpha-helix hence rationalizing the observed behavior.

\section{Conclusions.}


A joint application of experimental and computational approaches has revealed the rather high attitude of crabrolin of adopting alpha-helix conformation not only in membrane-mimicking solvents but also in the presence of relatively high concentration of water. Such behavior could enhance the peptide-membrane interaction which ultimately can lead to membrane leakage with final bacterial lysis. More conclusive answers might be obtained by simulations in the presence of the membrane. However quantitative treatment of conformational analysis in membrane might be frustrated by the difficulty in reaching an actual equilibrated condition.

Our study shows that this propensity essentially resides in the intrinsic thermodynamic stability of alpha-helix conformation whose kinetic stability, still high in TFE, is rather low in pure water. On the whole, our results demonstrate that crabrolin could be included in the family of the natural peptides potentially able to counteract infectious microorganisms otherwise resistant to conventional antibiotics and, hence, deserves additional investigations aimed to improve its antibacterial activity and to decrease the hemolytic effect.

\section{References}

1. A. C. Rinaldi. Curr. Opin. Chem. Biol. 2002, 6, 799.

2. M. Zasloff Nature 2002, 415, 389.

3. K.L. Brown, R.E.W. Hancock Curr. Opin. Immunol. 2006, 18, 24-30.

4. K. Radek, G. Gallo Semin. Immunopathol. 2007, 29, 27.

5. R.E.W. Hancock, H.-G. Sahl, Nat. Biotechnol. 2006, 24, 1551. 
6. R. Jelinek, S. Kolusheva. Curr. Prot. Pept. Sci. 2005, 6, 103.

7. Y. Shai Biopolymers 2002, 66, 236.

8. H.W. Huang, F.-Y. Chen, M.-T. Lee Phys. Rev. Lett. 2004, 24, 198304/1.

9. K. Lohner, S.E. Blondelle Comb. Chem. High Throughput Screen 2005, 8, 241.

10. T. Salditt, C. Li, A. Spaar Biochim. Biophys. Acta 2006, 1758, 1483.

11. A. Mecke, D.-K. Lee, A. Ramamoorthy, B.G. Orr, M.M.B. Holl Biophys. J. 2005; $89,4043$.

12. M. D’Abramo, A.C. Rinaldi, A. Bozzi, A. Amadei, G. Mignogna, A. Di Nola, M.. Aschi, Biopolymers 2006, 81, 215.

13. C. Coccia, A.C. Rinaldi, V. Luca, D. Barra, A. Bozzi, A. Di Giulio, E.C. Veerman, M.L. Mangoni Eur. Biophys. J. 2011, 40, 577.

14. G. Maisetta, A. Vitali, M.A. Scorciapino, A.C. Rinaldi, R. Petruzzelli, F.L., Brancatisano, S. Esin, A. Stringaro, M. Colone, C. Luzi, A. Bozzi, M. Campa, G. Batoni, FEBS J.2013, 280, 2842.

15. A. Di Gianpaolo, C. Luzi, B. Casciaro, A. Bozzi, M.L. Mangoni, M. Aschi, Biopolymers 2014, 102, 159.

16. M. Aschi, C. Luzi, A. Fiorillo, A. Bozzi Biopolymers 2015, 103, 692.

17. A. Argiolas, J.J. Pisano J. Biol. Chem. 1984, 259 (16), 10106. 
18. F. Delaglio, S. Grzesiek, G.W. Vuister, G. Zhu, J. Pfeifer, A. Bax, J. Biomol. NMR 1995, 6, 277.

19. T. D. Goddard, D.G. Kneller SPARKY 3, University of California, San Francisco CA. 2008

20. W. Lee, M. Tonelli, J. L. Markley Bioinformatics 2015, 31, 1325.

21. P. Güntert, L. Buchner J. Biomol. NMR 2015, 62, 453.

22. E.F. Pettersen, T.D. Goddard, C.C. Huang, G.S. Couch, D.M. Greenblatt, E.C. Meng, T.E. Ferrin, J. Comput. Chem. 2004, 25, 1605.

23. R. Koradi, M. Billeter, K. Wüthrich, K. J. Mol. Graph. 1996, 14: 29-32.

24. D. Van Der Spoel, E. Lindahl, B. Hess, G. Groenhof, A.E. Mark, H.J.C. Berendsen J. Comput. Chem. 2005, 26, 1701.

25. H.J.C. Berendsen, J.P.M. Postma, W.F. van Gunsteren, J. Hermans, In: Pullman B. editor. Intermolecular Forces, Dordrecht: Reidel; 1981, p. 331-342.

26. A.R. Van Buuren, H.J.C. Berendsen Biopolymers 1993, 33, 1159. 
27. G. Bussi, D. Donadio and M. Parrinello, J. Chem. Phys., 2007, 126, 014101.

28. W.L. Jorgensen, D.S. Maxwell, J. Tirado-Rives J. Am. Chem. Soc. 1996, 118, 11225.

29. B. Hess, H. Bekker, H.J.C. Berendsen, J.C.E.M. Frajie J. Comput. Chem. 1997; $18,1463$.

30. T.A. Darden, D.M. York, L.G. Pedersen J. Chem. Phys. 1993, 98, 10089.

31. A. Amadei, A.B.M. Linssen H.J.C. Berendsen Proteins: Struct. Funct. Genet. $1993 ; 17,412$.

32. P. Luo and R. L. Badwin , 1997, Biochemistry, 36, 8413-8421

33. O. Crescenzi, R. Guerrini, D. Picone, S. Salvadori, T. Tancredi P.A. Temussi Biopolymers 2000, 53, 257.

34. K. Wuthrich NMR of proteins and nucleic acids. New York: Wiley; 1986.

35. I. Daidone, A. Amadei, A. WIREs: Comput. Mol. Sci. 2012, 2, 762.

36. F. Eisenhaber, P. Lijnzaad, P. Argos, C. Sander, M. Scharf J. Comput. Chem.1995, 16, 273. 
37. M. D'Alessando, A. Amadei, M. Stener, M. Aschi J. Comput. Chem. 2015, 36, 399.

38. V. Krishnakumari, R. Nagaraj. J. Peptide Res. 1997, 50, 88.

\section{Acknowledgements}

This work has been supported by iNEXT, project number 653706, funded by the Horizon 2020 programme of the European Union. We also acknowledge CINECA (Italy) for the project IsCa4-AUPS (ISCRA—class C).

We acknowledge Prof. Pierandrea Temussi and Dr. Andrea Amadei for stimulating discussions and Mr. Renato Di Bartolomeo (University of L'Aquila) for his help in the preparation of the manuscript. 